\title{
Integrating Lean and Agile Strategies into the Production Control System for Mixed- model Production Lines
}

\author{
Katsuhiko Takahashi, Kana Yokoyama and Katsumi Morikawa \\ Department of Artificial Complex Systems Engineering, \\ Graduate School of Engineering, Hiroshima University \\ 1-4-1, Kagamiyama, Higashi-Hiroshima, 739-8527, Japan. \\ \{takahasi,k4580,mkatsumi\}@hiroshima-u.ac.jp \\ WWW home page: http://www.pel.sys.hiroshima-u.ac.jp
}

\begin{abstract}
For mixed-model production lines, this paper proposes a production control system based on lean and agile strategies for responding to changes in product mix is proposed. Performance of the proposed system under the conditions of unstable changes in product-mix is analyzed by simulation experiments, and it can be claimed that the proposed system can respond to changes in product mix by re-allocating work elements into each work center, and inventories can be decreased without decreasing customer service level.
\end{abstract}

Keywords

Lean production, Agile production, Production control, Mixed-model line

\section{Introduction}

Based on the diversification of customers needs, recent production environments become more and more uncertain and competitive. Under the uncertain and competitive production environments, production lines for mass production are requested not only to produce efficiently but also adjust their own systems effectively, that is not only lean but also agile production is requested in the production lines. Then, production lines have to be shifted from single model lines to mixed-model lines, and mass-production has to be shifted to mass-customization. Furthermore, in order to realize mass-customization, mixed-model production lines are requested to have a function to respond to changes in product-mix.

In the previous literature on production control systems for single-model production lines, systems based on lean and agile strategies have been proposed 
(Hopp and Roof [1], Takahashi and Nakamura [2]). In the systems, just-in-time (JIT) ordering based on lean strategy is included, and the function to detect unstable changes in demand and adjust the buffer size, that is based on agile strategy, is also included. However, in the previous literature on mixed-model production lines, line balancing problems have been considered by various researchers only from the view point of lean strategy, and there is no literature on the problems from the view point of not only lean but also agile strategies. In order to integrate agile strategy into the mixed-model production lines, not only adjusting the buffer size but also reallocating the work elements into each work center are necessary to be considered in responding to changes in product-mix.

Therefore, in this paper, a production control system based on lean and agile strategies is proposed for responding to changes in product mix through re-allocating work elements into each work center, and the performance under the conditions of unstable changes in product-mix is analyzed by simulation experiments.

\section{Mixed-model Production Lines}

In this section, the mixed-model production line considered in this paper and CONWIP control for the production line are defined.

\subsection{Assumptions}

The mixed-model production line considered in this paper is assumed as follows:

(1) The production line produces multiple standard products that can be made to stock.

(2) The demand for product fluctuates stochastically, and it includes stable and unstable changes. The mean $\mu_{D}$ and the standard deviation $\sigma_{D}$ of total demand per time are fixed, but the product-mix, the demand ratio of product $j, r_{j}$, fluctuates not only stably but also unstably. Let $d_{i, j}$ be the $i$ th inter-arrival time of product $j$, the mean $\mu_{i, j}$ fluctuates not only stably but also unstably, but the standard deviation $\sigma_{i, j}$ is fixed. A backorder of demand can be allowed.

(3) All of the products are produced through a serial production line with $N$ stages, and each stage has a work center called the 1 st, 2 nd, or $N$ th stage accordingly, as the process proceeds. The number of stages, $N$, is fixed.

(4) The process at each work center is un-paced, and the process time fluctuates stochastically. Work elements are allocated to each work center, and the mean $\mu_{P}^{(j, n)}$ and standard deviation $\sigma_{P}^{(j, n)}$ are obtained from the process time of allocated work elements. The allocated work elements are re-allocated in response to unstable changes in product-mix.

(5) Each stage has an inventory point for stocking the items processed at the stage, and the buffer inventory is stocked at the inventory point of the final stage. The buffer size $S_{j}$ of item $j$ is controlled dynamically in response to unstable changes in product-mix. 


\subsection{CONWIP Control}

For the production line defined above, a production control system is proposed by integrating lean and agile strategies. In the proposed production control system, constant work-in-process (CONWIP) control [3] is utilized as an alternative to kanban control in just-in-time ordering, that is based on lean strategy, and the CONWIP control is useful to modify the allocation of work elements at each work center (Takahashi and Morikawa [4]).

In the CONWIP control, after releasing the orders of the pre-determined quantities for all products, a new order of a product is released to the first stage just after the product has been completed. Then, at each stage, each order is processed based on first in first out (FIFO) policy.

Based on the CONWIP control, the $i$ th order release time $O_{i, j}{ }^{(n)}$ of product $j$ to stage $n$ can be formulated as follows:

$$
\begin{aligned}
& O_{i, j}^{(1)}=\max \left\{D_{i, j}, P_{\left.i-S_{j, j}^{(N)}\right\}}\right. \\
& O_{i, j}^{(n)}=\max \left\{P_{i, j}^{(n-1)}, \max _{\{k, l) \in Q_{i, j}^{(n)}} P_{k . l}^{(n)}\right\} \quad(n=2,3, \cdots, N)
\end{aligned}
$$

Here, $D_{i, j}$ is the $i$ th arrival time of product $j$, that is $D_{i, j}=D_{i-1, j}+d_{i, j}$, and the set of previous order release times $Q_{i, j}{ }^{(n)}$ is formulated as follows:

$$
\begin{aligned}
& Q_{i, j}^{(1)}=\left\{(k, l) \mid O_{k, l}^{(1)} \leq O_{i, j}^{(1)}\right\} \\
& Q_{i, j}^{(n)}=\left\{(k, l) \mid O_{k, l}^{(n)} \leq P_{i, j}^{(n-1)}\right\} \quad(n=2,3, \cdots, N)
\end{aligned}
$$

Also, $P_{i, j}^{(n)}$ is the $i$ th completion time of the production of product $j$ at stage $n$, and it can be formulated as follows:

$$
\begin{aligned}
& P_{i, j}^{(1)}=\max \left\{O_{i, j}^{(1)}, \max _{(k, l) \in Q_{i, j}^{(1)}} P_{k, l}^{(1)}\right\}+p_{i, j}^{(1)} \\
& P_{i, j}^{(n)}=\max \left\{O_{i, j}^{(n)}, P_{i, j}^{(n-1)}, \max _{(k, l) \in Q_{i, i}^{(n)}} P_{k . l}^{(n)}\right\}+p_{i, j}^{(n)} \quad(n=2,3, \cdots, N)
\end{aligned}
$$

Here, $p_{i, j}^{(n)}$ is the $i$ th process time of the production of product $j$ at stage $n$.

\section{Analyzing the Effects of Product-Mix}

The performance of the mixed-model production line defined above is analyzed by simulation experiments, and the effects of product-mix are investigated in this section.

\subsection{Experimental Conditions}

In this section, the simulation experiments under the following conditions with stable demand are planned.

(1) Two products are produced in the production line.

(2) The inter-arrival time of product demand is assumed to be a normally distributed variable with mean $\mu_{D}=1.05$ and standard deviation $\sigma_{D}=0.05$, and the demand ratio between products, $r=0.0,0.005,0.010, \ldots, 1.0$.

(3) For producing products, 15 work elements with mean process time and 
Table 1. Work elements, immediately preceding elements and mean process time

\begin{tabular}{|c|c|c|c|c|c|c|c|c|c|c|c|c|c|}
\hline \multirow{3}{*}{ 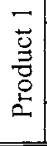 } & element & 1 & 3 & 4 & 5 & 6 & 8 & 9 & 10 & 13 & 14 & 15 & \\
\hline & $\begin{array}{c}\text { immediately } \\
\text { preceding }\end{array}$ & - & 1 & 1 & 1 & 1 & 3 & 3 & $\begin{array}{l}4,5 \\
6 \\
\end{array}$ & 8,9 & 14 & $\begin{array}{l}13, \\
14\end{array}$ & \\
\hline & process time & 0.6 & 0.2 & 0.5 & 0.7 & 0.1 & 0.2 & 0.6 & 0.3 & 0.5 & 0.5 & 0.1 & \\
\hline \multirow{3}{*}{ 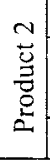 } & element & 1 & 2 & 4 & 5 & 6 & 7 & 10 & 11 & 12 & 13 & 14 & 15 \\
\hline & $\begin{array}{c}\text { immediately } \\
\text { preceding }\end{array}$ & - & - & 1 & 1 & 1 & 2 & $\begin{array}{l}4,5 \\
6,7 \\
\end{array}$ & 7 & 7 & $\begin{array}{l}11, \\
12 \\
\end{array}$ & 10 & $\begin{array}{l}13, \\
14\end{array}$ \\
\hline & process time & 0.6 & 0.3 & 0.5 & 0.7 & 0.1 & 0.4 & 0.3 & 0.3 & 0.2 & 0.5 & 0.5 & 0.4 \\
\hline
\end{tabular}

Table 2. Allocated work elements, $\psi$, and mean process time $\mu_{P}^{(j, n)}$ of product $j$ at stage $n$ based on the demand ratio $r$

\begin{tabular}{|c|c|c|c|c|c|c|}
\hline Allocation $\psi$ & \multicolumn{3}{|c|}{1} & \multicolumn{3}{|c|}{2} \\
\hline Ratio $r$ & \multicolumn{3}{|c|}{$[0.0,0.20]$} & \multicolumn{3}{|c|}{$(0.20,0.33]$} \\
\hline Stage $n$ & elements & $\mu_{p}^{(1, n)}$ & $\mu_{P}^{(2, n)}$ & elements & $\mu_{P}^{(1, n)}$ & $\mu_{P}^{(2, n)}$ \\
\hline 1 & $1,2,6$ & 0.7 & 1.0 & $1,2,3,6$ & 0.9 & 1.0 \\
\hline 2 & $3,4,7,9$ & 1.3 & 0.9 & $4,7,9$ & 1.1 & 0.9 \\
\hline 3 & 5,11 & 0.7 & 1.0 & 5,11 & 0.7 & 1.0 \\
\hline 4 & $10,12,14$ & 0.8 & 1.0 & $10,12,14$ & 0.8 & 1.0 \\
\hline 5 & $8,13,15$ & 1.1 & 0.9 & $8,13,15$ & 1.1 & 0.9 \\
\hline Allocation $\psi$ & \multicolumn{3}{|c|}{3} & \multicolumn{3}{|c|}{ 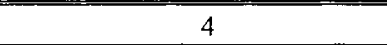 } \\
\hline Ratio $r$ & \multicolumn{3}{|c|}{$(0.33,0.42]$} & \multicolumn{3}{|c|}{$(0.42,0.50]$} \\
\hline Stage $n$ & elements & $\mu_{P}^{(1, n)}$ & $\mu_{P}^{(2, n)}$ & elements & $\mu_{p}{ }^{(1, n)}$ & $\mu_{P}^{(2, n)}$ \\
\hline 1 & $1,2,3,6$ & 0.9 & 1.0 & $1,2,3,8$ & 1.0 & 0.9 \\
\hline 2 & $4,7,11$ & 0.5 & 1.2 & $4,6,7,12$ & 0.6 & 1.2 \\
\hline 3 & 5,9 & 1.3 & 0.7 & 5,9 & 1.3 & 0.7 \\
\hline 4 & $10,12,14$ & 0.8 & 1.0 & $10,11,13$ & 0.8 & 1.1 \\
\hline 5 & $8,13,15$ & 1.1 & 0.9 & 14,15 & 0.9 & 0.9 \\
\hline Allocation $\psi$ & \multicolumn{3}{|c|}{5} & \multicolumn{3}{|c|}{6} \\
\hline Ratio $r$ & \multicolumn{3}{|c|}{$(0.50,0.64]$} & \multicolumn{3}{|c|}{$(0.64,0.72]$} \\
\hline Stage $n$ & elements & $\mu_{P}^{(1, n)}$ & $\mu_{p}^{(2, n)}$ & elements & $\mu_{P}^{(1 . n)}$ & $\mu_{P}^{(2, n)}$ \\
\hline 1 & $1,2,3,6$ & 0.9 & 1.0 & $1,2,3,6$ & 0.9 & 1.0 \\
\hline 2 & $4,8,9$ & 1.3 & 0.5 & 4,9 & 1.1 & 0.5 \\
\hline 3 & $5,7,12$ & 0.7 & 1.3 & $7,8,11,12,13$ & 0.7 & 1.4 \\
\hline 4 & $10,11,13$ & 0.8 & 1.1 & 5,10 & 1.0 & 1.0 \\
\hline 5 & 14,15 & 0.9 & 0.9 & 14,15 & 0.9 & 0.9 \\
\hline Allocation $\psi$ & \multicolumn{3}{|c|}{7} & \multicolumn{3}{|c|}{8} \\
\hline Ratio $r$ & \multicolumn{3}{|c|}{$(0.72,0.83]$} & \multicolumn{3}{|c|}{$(0.83,1.0]$} \\
\hline Stage $n$ & elements & $\mu_{P}^{(1, n)}$ & $\mu_{P}^{(2, n)}$ & elements & $\mu_{p}^{(1, n)}$ & $\mu_{P}^{(2, n)}$ \\
\hline 1 & $1,2,8$ & 1.0 & 0.6 & $1-3,6$ & 0.9 & 1.0 \\
\hline 2 & 4,9 & 1.1 & 0.5 & $7,8,9,11,12$ & 0.8 & 0.9 \\
\hline 3 & $2,5,6,7$ & 0.8 & 1.5 & 4,13 & 1.0 & 1.0 \\
\hline 4 & $10,11,12,13$ & 0.8 & 1.3 & 5,10 & 1.0 & 1.0 \\
\hline 5 & 14,15 & 0.9 & 0.9 & 14,15 & 0.9 & 0.9 \\
\hline
\end{tabular}


precedence relationship are assumed as shown in Table I. Also, the process time of each work element is assumed to be a normally distributed variable with the coefficient of variation 0.15 and independent with each other.

(4) Work elements shown in Table 1 are allocated to 5 stages in order to make weighted sum of the mean process times with the demand ratio less than the cycle time 10 by line balancing, and the resulted allocations according to the demand ratio are shown in Table 2.

(5) Buffer size of each product is assumed as $S_{j}=1,2, \ldots, 10,(j=1,2)$.

(6) A simulation run-length is 100,000 time units, excluding the warm-up run of 5,000 time units, and the number of replications for each simulation run is one.

(7) As performance measures, the mean waiting time of demand, wt, and the total of mean work-in-process inventories, twip, are evaluated.

\subsection{Results and Implications}

Under the experimental conditions shown above, simulation experiments are performed, and the results are shown in Fig. 1.
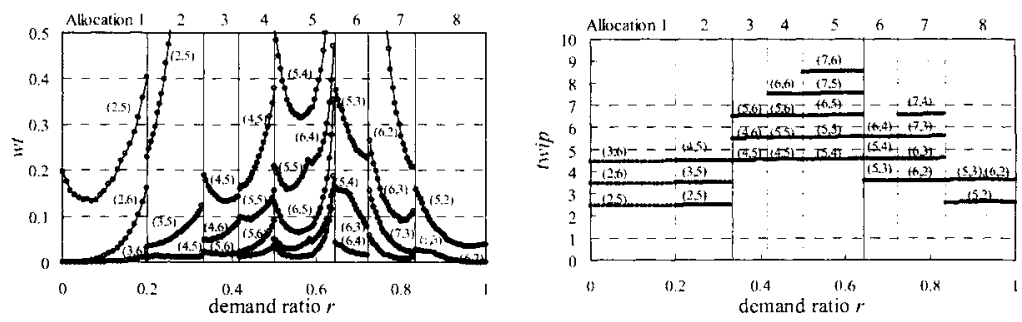

Fig. 1. The effects of the demand ratio, $r$, the allocation of work elements, $\psi$, and buffer sizes, $\left(S_{1}, S_{2}\right)$, on the two performance measures, wt and twip

With Fig. 1, it can be seen that $w t$ increases as $r$ increases from 0 to 0.5 or decreases from 0.5 to 1.0 if the allocation of work elements and buffer size are fixed at a certain level. At the same time, twip does not change even if $r$ changes. It can be stated that, if $r$ changes as an unstable change and the allocation of work elements and buffer size are fixed, wt suffers from a significant influence of the unstable change. However, with Fig. 1, it can be seen that wt decreases whereas twip increases, as the allocation of work elements changes or the buffer size increases. Therefore, in order to minimize twip while maintaining $w t$ at less than a certain level, the allocation of work elements and/or the buffer size should be altered in response to the changed $r$. In responding to the changed $r$, altering only the buffer size is insufficient, but the allocation of work elements should be also altered. For example, in order to minimize twip while maintaining $w t<0.20$, the buffer size should be altered from $(2,5)$ to $(2,6)$ in response to changes in $r$ from $[0,1.4)$ to $[1.4,0.2]$ under allocation 1. In order to respond to changes in $r$ as $r>0.2$, it is difficult to maintain $w t<0.2$ even if the buffer size is altered. In the case, the allocation of work elements should be altered from allocation 1 to 2 , and the buffer size is also altered in response to changes in $r$. 


\section{Proposed Production Control System}

Based on the effects of product-mix analyzed above, a lean and agile system that can detect unstable changes in product-mix and control the allocation of work elements and buffer size as a reaction to the detected unstable changes is proposed in this section.

\subsection{Detecting Unstable Changes}

In order to react to unstable changes in product-mix, the time series of the interarrival time of demand is monitored, and only unstable changes must be detected from the time series data. For the purpose, control charts are utilized in process control. Various control charts have been developed, and the EWMA chart [5] is utilized to detect small changes quickly. In this paper, the EWMA chart is utilized to detect unstable changes in demand like Takahashi and Nakamura [2]. At first, the monitored time series data $d_{i, j}$ are filtered by exponential smoothing, a kind of lowpass filter, and the EWMA $H_{i, j}$ are calculated as follows;

$$
H_{i, j}=\alpha_{j} d_{i, j}+\left(1-\alpha_{j}\right) H_{i-1, j}
$$

where $\alpha_{j}$ is the smoothing constant.

Based on the filtered data of inter-arrival time, the demand ratio can be calculated as $r=H_{i, 2} / H_{i, 1}$. However, in this paper, the EWMA $H_{i, j}$ are compared with the upper and lower control limits, and unstable changes are detected whenever any of the data is out of these limits. Based on the steady-state mean and variance, the upper and lower control limits, $L C L_{c, j}, U C L_{c, j}$ to detect the unstable change in demand ratio for which the current allocation of work elements $\psi_{c}$ and buffer size $S_{c}=\left(S_{j}\right)$ should be altered can be formulated as follows;

$$
\begin{aligned}
U C L_{c, j} & =\bar{\mu}_{,}^{(i)}+\gamma_{i} \sqrt{\frac{\alpha_{j}}{2-\alpha_{i}}} \sigma_{D}^{(j)} \\
U C L_{c, j} & =\bar{\mu}_{c}^{(j)}-\gamma_{j} \sqrt{\frac{\alpha_{j}}{2-\alpha_{j}}} \sigma_{D}^{(j)}
\end{aligned}
$$

where $\gamma_{j}$ is a parameter of allowance. Also, $\bar{\mu}_{c}^{(j)}$ and $\underline{\mu}^{(j)}$ are the upper and lower limits of the interval of demand of product $j$ for which the current allocation $\psi_{c}$ and the buffer size $S_{c}$ under the condition of the allocation are appropriate, and the limits are obtained from the simulation results under the stable-demand conditions above.

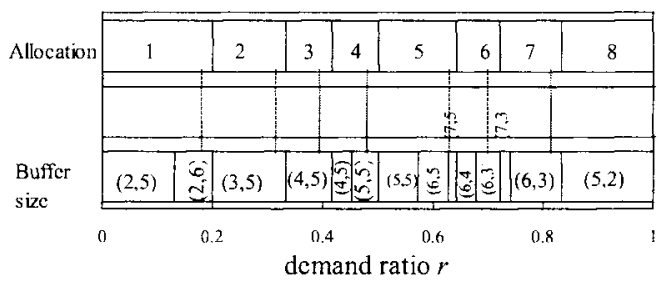

Fig. 2. The allocation of work elements and buffer size $(\psi, S)$ to minimize twip while maintaining $w t<0.2$ in response to the demand ratio $r$ 
For example, the allocation of work elements and buffer size $(\psi, S)$ to minimize twip while maintaining $w t<0.2$ in response to the demand ratio are obtained as shown in Fig. 2. Obtained interval of demand ratio can be translated into the interval of demand inter-arrival time of each product.

\subsection{Adjusting WIP Level and Re-allocating Work Elements}

If an unstable change is detected, the current allocation of work elements and/or buffer size must be altered in response to the detected change. For the purpose, the control rule for modifying the allocation of work elements and buffer sizes must be developed. The relationship between demand ratio $r$ and the appropriate allocation of work elements and buffer size shown in Fig. 1 can be utilized, and Fig. 2 shows the allocation and buffer sizes to minimize twip while maintaining $w t<0.2$.

Based on the relationship in Fig. 2, the allocation of work elements and buffer sizes can be controlled in response to unstable changes in demand ratio $r$. When an unstable change in demand ratio $r$ is detected by the limits, the allocation of work elements and buffer size are altered from the current ones $\left(\psi_{c}, S_{c}\right)$ as follows:

$$
(\psi, S)=\left\{\begin{array}{cc}
\left(\psi_{c}, S_{c}\right) & \left(\forall j, L C L_{j}<H_{i, j}<U C L_{j}\right) \\
\left(\psi_{m}, S_{m}\right) & \text { (otherwise })
\end{array}\right.
$$

Here, the modified allocation and buffer size $\left(\psi_{m}, S_{m}\right)$ is obtained from the interval of the demand ratio in which the demand ratio calculated from the exceeded EWMA $H_{i, j}$ is included.

\section{Investigating Effectiveness of the Proposed System}

Effectiveness of the proposed system is investigated and compared with those of the alternative system based on only lean strategy. In the investigation, no delay in detecting unstable changes in demand and modifying the allocation of work elements and buffer sizes is assumed. Under the assumption, performance of the proposed system can be expected from the simulation results under stable demand condition shown in section 3, and the obtained results are shown in Fig. 3.

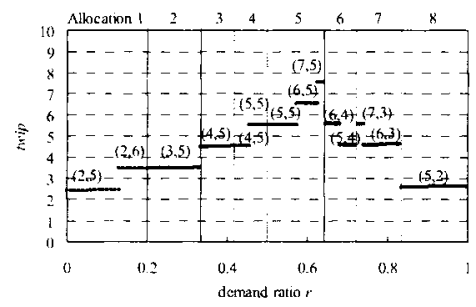

Fig. 3. The expected twip of the proposed system for maintaining $w t<0.2$ 
With Fig. 3, we can calculate the average of the expected twip for demand ratio $0.0<r<1.0$. The obtained average is 4.16 , and it is about $8.4 \%$ less than that of the fixed allocation (allocation 8 ) and fixed buffer sizes $(5,4)$, that is the best in utilizing only lean strategy. The simulation results show that the proposed production control system based on lean and agile strategies can decrease the total of mean work-inprocess inventory without increasing the mean waiting time of demand, and it can be claimed that the proposed production control system can respond to unstable changes in product-mix effectively.

\section{Conclusions}

Under the recent uncertain and competitive production environments, lean and agile production lines with the production control system proposed in this paper are valuable for achieving a practical goal to decrease inventories without decreasing customer service level. Then, it can be claimed that the proposed production control system is practically valuable.

In the mixed-product production line in this paper, the allocation of work elements to each work center is assumed to be able to modify in response to changes in product-mix. Therefore, multi-skilled workers or highly functional work centers are assumed implicitly. However, the fixed mean process time of each work element is assumed, and the learning effects of process time have not yet considered. Also, the mean and variance of the total demand are assumed to be fixed and changes in the mean and variance of demand have never been considered in this paper. If changes not only in product-mix but also in the mean and variance of demand are considered, the number of work centers as well as the re-allocation of work elements should be changed in response to the detected changes.

\section{Acknowledgements}

The work in this paper was partially supported by the Grant-in-Aid for scientific research in Japan Society for the Promotion of Science in 2006-2008.

\section{References}

1. W. J. Hopp and M. L., Roof, Setting WIP Levels with Statistical Throughput Control (STC) in CONWIP Production Lines, IJPR, 36(4), 867-882, (1998).

2. K. Takahashi and N. Nakamura, Reacting JIT Ordering Systems to the Unstable Changes in Demand, IJPR, 37(10), 2293-2313, (1999).

3. M. L. Spearman, D. L. Woodruff, and W. L. Hopp, CONWIP: A pull alternative to kanban, IJPR, 28(5), 879-894, (1990).

4. K. Takahashi and K. Morikawa, A System for Monitoring and Supervising Reconfigurable Manufacturing Systems, Proc. 7th Int'l Conf. Monitoring \& Automatic Supervision in Manufacturing, 1-8, (2004).

5. J. M. Lucas and M. S. Saccucci, Exponentially Weighted Moving Average Control Schemes: Properties and Enhancements, Technometrics, 32(1), 1-12, (1990). 